\title{
Ambientes digitales de aprendizaje en educación a distancia para la formación inicial de docentes: percepciones acerca de su pertinencia
}

\section{Digital environments of learning in distance education for the initial education of teachers: perceptions about their relevance}

\author{
José Julián Ñáñez-Rodríguez1 \\ Juan Carlos Solano-Guerrero2 \\ Edwin Bernal-Castillo3 \\ Recibido: abril 03 de 2019 \\ Aceptado: junio 28 de 2019
}

\section{Resumen}

En este documento se da una mirada a la percepción de docentes y estudiantes sobre implementación de Tecnologías de Información y Comunicación, TIC, junto con la experiencia de los portafolios pedagógicos, en programas de licenciatura de una universidad colombiana. El trabajo tuvo un enfoque cualitativo, de carácter exploratorio y correlacional, del tipo investigación acción. La muestra estuvo conformada por 77 docentes y 192 estudiantes, de la Universidad del Tolima. Los estudiantes presentan una actitud positiva ante las TIC, la cual está fuertemente correlacionada con su experticia en esas tecnologías. El uso de TIC por parte de los docentes, se ve menguado en la medida en que ellos, según la percepción de los estudiantes, dan mayor relevancia al trabajo orientado presencialmente. La percepción de los estudiantes fue positiva con respecto al portafolio pedagógico, considerándolo como una herramienta que contribuye a la formación y el aprendizaje autónomo, dos aspectos esenciales

\begin{abstract}
This document looks at the perception of teachers and students on the implementation of Information and Communication Technologies, ICT, together with the experience of the pedagogical portfolios, in degree programs of Colombian universities. The work had a qualitative, exploratory and correlational approach, of the action research type. The sample consisted of 77 teachers and 192 students, from the Universidad del Tolima. Students have a positive attitude towards ICT, which is strongly correlated with their expertise in these technologies. The use of ICT by teachers is diminished to the extent that they give more relevance to face-to-face work, according to the students' perception. The students' perception was positive with respect to the pedagogical portfolio, considering it as a tool that contributes to formation and autonomous learning, two essential aspects of distance education. It is concluded that the educative processes transcend the instructional and training exercises, while they seek a transformation of the
\end{abstract}

1 Licenciado en Filosofía y Educación Religiosa, Doctor en Ciencias de la Educación, Universidad del Tolima, Ibagué, Colombia. E-mail: jjnanezr@ut.edu.co ORCID: 0000-0002-1221-7050

2 Ingeniero de Sistemas, Magíster en Tecnologías de la Información Aplicadas a la Educación, Universidad del Tolima, Ibagué, Colombia. E-mail: jcsolano@ut.edu.co ORCID: 0000-0002-2222-6954

3 Licenciado en Matemáticas con Énfasis en Informática, Magíster en Tecnologías de la Información Aplicadas a la Educación, Universidad del Tolima, Ibagué, Colombia. E-mail: ebernalc@ut.edu.co ORCID: 0000-0001-8073-9347 
de la educación a distancia. Se concluye que los procesos de formación trascienden los ejercicios instructivos y de capacitación, en tanto que pretenden una transformación del sujeto, que trasciende la lógica de la ejecución procedimental.

Palabras clave: contexto de aprendizaje, formador de docentes, tecnología educativa, material pedagógico, educación a distancia.

\section{Introducción}

Las nuevas condiciones sociales, culturales, políticas, económicas y tecnológicas plantean exigencias de transformación a la educación colombiana, que priorizan procesos de cambio en las instituciones de educación superior (Cruz-Rojas, Molina-Blandón \& Valdiri-Vinasco, 2019). Tales cambios se evidencian en el currículo de los programas de formación, desde los cuales se requiere generar transformaciones en la cultura académica. La Universidad del Tolima (2015), con más de 30 años de experiencia en educación a distancia, supone retos en este sentido que obligan a transformar la modalidad en sí misma. Esto implica una mirada a las concepciones y planteamientos curriculares, así como a la integración que se hace de las Tecnologías de Información y Comunicación, TIC, en los ambientes de aprendizaje.

La Formación Inicial Docente, FID, no es ajena a estas circunstancias, pues las mediaciones tecnológicas se constituyen en un eje estratégico de la misma, en sus procesos pedagógicos y didácticos. Las experiencias de aprendizaje que incorporan TIC, a las que se enfrentan las y los profesores en formación, influyen, si no definitivamente, sí de manera importante sus posteriores prácticas profesionales. Tal situación cobra una dimensión más compleja si se reconoce que el profesor es responsable, en gran medida, de agenciar procesos de subject, which transcends the logic of procedural execution.

Keywords: learning environment, teacher educators, educational technology, teaching materials, distance education.

reproducción, creación o transformación cultural de las instituciones escolares.

La Universidad del Tolima (2015), se orienta hacia una formación flexible, considerando en sus lineamientos y políticas, diferentes factores asociados a la flexibilidad, entre ellos, los tecnológicos. Esta formación requiere de transformaciones en la pedagogía y la didáctica universitarias, como la incorporación de las TIC enfocadas hacia los procesos educativos. La adaptación a los cambios científicos y tecnológicos en términos de su acceso, asimilación y proyección, compromete a las/os profesoras/es, quienes orientan y facilitan el proceso formativo de sus estudiantes.

La Institución ha ido incorporando las TIC en los procesos de formación, investigación, proyección social y de gestión. Tal incorporación se ha venido produciendo de diversas maneras, por parte de los programas de formación. El Proyecto Educativo Institucional, PEl, de la Universidad del Tolima (2015), ha formulado la política académica: educación mediada por TIC. Sin embargo, no existen lineamientos, planes y programas institucionales que articulen las acciones generadas por los programas de formación e integren las dimensiones organizativas, tecnológicas, educativas y comunicativas que ello implica y que direccionen sus alcances en las transformaciones curriculares. 
En ese sentido, en la FID de la modalidad de educación a distancia de la Universidad del Tolima, es escasa la apropiación y uso de ambientes digitales de aprendizaje, considerando la necesidad de desarrollar procesos más flexibles de enseñanza y aprendizaje. Para abordar este problema, se planteó la pregunta: ¿Cómo apropiar y usar ambientes digitales de aprendizaje en la formación inicial docente en la modalidad de educación a distancia, de la Universidad del Tolima?.

\section{Marco teórico y metodología}

\subsection{Formación inicial docente y nuevas tecnolo- gías}

Las TIC en la formación docente constituye un ámbito de investigación reciente en América Latina (Vaillant, 2013; Hepp, Marés \& Severin, 2012; Rozo-Sandoval \& Prada-Dussán, 2012; Salinas, 2012; Brun, 2011), señalándose que: "en líneas generales, estos estudios coinciden en que la situación no es auspiciosa, lo que se asocia no solamente a un problema regional sino que constituye una tendencia internacional" (Dussel, 2014, p. 16). En cuanto a las tendencias en formación para la innovación educativa en el contexto colombiano, se plantea que: "La vinculación de [TIC] a los procesos de [FID], es considerada una de las problemáticas más representativas en la caracterización de la situación de la formación en Colombia" (Ministerio de Educación Nacional, 2013).

Dos de las dimensiones de la integración de TIC en FID, relacionadas con el presente estudio, se orientan a la integración curricular de TIC, de una parte, y de otra, al uso y apropiación de los recursos y herramientas educativas digitales por parte de los formadores de formadores. En cada dimensión se revisaron algunos estudios cualitativos, cuantitativos o mixtos, con predominancia de los primeros, con alcance exploratorio, descriptivo o interpretativo.

En la primera dimensión, se revisaron los estudios de: Gisbert-Cervera, González-Martínez y Este-
ve-Mon (2016); Leite, Martínez y Monteiro (2016); Álvarez (2015); Ballesta y Céspedes (2015); Gewerc y Montero (2015); Sancho, Bosco, Alonso y Sánchez (2015); Cózar y Roblizo (2014); Occelli, García y Masullo (2012); Silva-Quiroz y Astudillo-Cavieres (2012). Se identifican dos ejes: el primero, de procesos de incorporación de TIC en el currículo de la FID, y el segundo, de las competencias y los estándares TIC para la FID. Estos estudios destacan la necesidad de superar la perspectiva instrumental que ha predominado en la integración de TIC en los procesos educativos, frente a la reflexión y la acción pedagógica que exigen los nuevos escenarios de la educación superior.

En la segunda dimensión, se revisaron los estudios de: Fraga y Gewerc (2015); Garnica-Hoz (2015); Hinojosa, Valdez, Servín y Perusquía (2015); Laura, Sosa y Almanza (2015); Álvarez y Morán (2014); Arias y Vanegas (2013); Cifuentes y Cerda (2013); Parra y Cardona (2013); Díez (2012); Sánchez y Morales (2012); Guisao-Gil (2011); Tobón, Arbeláez, Falcón y Bedoya (2010); Mellado (2010); Garrido (2009). Se identifican dos ejes: el primero, de las representaciones sobre el uso y la apropiación de las TIC en la FID, y el segundo, de las estrategias educativas implicadas en el uso y apropiación de las TIC en la FID. En tales estudios, se destaca la reflexión y la acción formativa que se derivan de cada uno de los entornos digitales en el proceso de enseñanza y aprendizaje, enmarcados en las transformaciones educativas que enfrenta la educación superior, en contextos específicos.

\subsection{Metodología}

Se planteó un estudio cualitativo, desde la investigación acción (Elliott, 2010), la cual propone el cambio y la transformación a partir de la reflexión. Se realiza con la participación de los implicados, a partir de un diagnóstico para: identificar los problemas, buscar sus causas, encontrar soluciones y aplicarlas. El análisis se efectúa en torno a la perspectiva de docentes y estudiantes (actitudes), sobre la incorporación de TIC en la enseñanza y el aprendizaje flexibles, junto con las percepciones 
sobre la incorporación de TIC que poseen los directivos de programas de licenciatura.

En la primera etapa, se caracterizó el componente de informática educativa en la Formación Inicial de Docentes, FID, de la modalidad de educación a distancia de la Universidad del Tolima. Asimismo, se indagó sobre la apropiación y el uso de las $\mathrm{TIC}$, junto con actitudes y percepciones frente a la enseñanza y aprendizaje flexibles y el uso de TIC en el proceso educativo. En la segunda etapa, se establecieron lineamientos para la construcción de ambientes digitales de aprendizaje y se generó una experiencia de diseño y desarrollo de dos portafolios pedagógicos. En la tercera etapa, se recuperó la experiencia generada con los docentes y el equipo interdisciplinario, valorando el diseño de los portafolios desde la perspectiva de los estudiantes. En la cuarta etapa se finalizó con la reflexión sobre el proceso.

La población docente estuvo conformada por 118 docentes de la Universidad del Tolima, en el Semestre B de 2016, de los programas de FID en modalidad de educación a distancia, Centro de Atención Tutorial, CAT Ibagué, así: Licenciatura en Ciencias Naturales y Educación Ambiental, Licenciatura en Educación Artística, Licenciatura en Literatura y Lengua Castellana, y Licenciatura en Pedagogía Infantil. La población estudiantil estuvo conformada por 1.356 estudiantes de la institución y programas antes mencionados.

En la aplicación del cuestionario a los docentes, se estimó la muestra en 80 , considerando un error del $5.5 \%$, una proporción de 0,5 y una confianza del $90 \%$, realizándose on line y off line, para lo cual se hizo difusión mediante redes sociales y correos electrónicos institucionales. Para la aplicación del cuestionario a los estudiantes, se estimó la muestra en 193, considerando un error del 5.5\%, una proporción de 0,5 y una confianza del $90 \%$. El cuestionario se aplicó on line y off line, para lo cual se hizo difusión mediante redes sociales y correos electrónicos institucionales.
Se adelantaron dos estudios de carácter exploratorio y descriptivo, de corte cualitativo. Uno sobre las percepciones respecto a las TIC y los niveles de apropiación de las mismas, aplicando entrevistas semiestructuradas (Valles, 2007), a dos docentes de cada programa. El análisis de las entrevistas se basó en los planteamientos sobre percepciones respecto a las TIC, de Riascos, quintero y Avila (2009), y sobre apropiación de TIC, de Hooper y Rieber (1995).

Para la segunda etapa, considerando la estrategia de formación en la modalidad de educación a distancia en la Universidad del Tolima, enfocada a la FID, y luego de una revisión en torno a los ambientes híbridos y los entornos digitales de aprendizaje, se plantearon criterios pedagógicos, comunicativos y tecnológicos para su construcción y aplicación, en el marco de un ciclo de vida. Teniendo en cuenta el compromiso de diseñar, desarrollar, integrar y validar ambientes digitales para dos cursos, se inició con el diseño y desarrollo de un taller de formación dirigido a docentes de las licenciaturas. Con los portafolios pedagógicos diseñados, resultado del taller, se dio paso a su producción con la participación del equipo interdisciplinar de la Unidad de Pedagogía y Mediaciones Tecnológicas del IDEAD.

En la tercera etapa, finalizada la construcción de portafolios pedagógicos, los docentes hicieron un ejercicio de narración abierta sobre su experiencia, lo que permitió identificar fortalezas y debilidades. Se utilizó esta técnica pues ofrece mayores posibilidades para que quien narra pueda presentar su vivencia, sin las restricciones que implican técnicas más estructuradas.

Asimismo, con el fin de valorar el diseño de los portafolios pedagógicos en los cursos Lenguaje, cognición y sociedad, de la Licenciatura en Literatura y Lengua Castellana, y Filosofía de la educación, de la Licenciatura en Educación Infantil, se asumieron los criterios propuestos como afirmaciones de un instrumento a partir de una escala Likert, complementado con preguntas abiertas 
sobre la experiencia. Se aplicó el instrumento a 22 estudiantes: 9 del primer curso (Grupo 1), y 13 del segundo (Grupo 2).

\section{Resultados y discusión}

3.1 Perspectivas de incorporación de TIC en la enseñanza y el aprendizaje flexibles

De los 80 docentes que respondieron las preguntas, el $42,5 \%$ son de género femenino y el $57,5 \%$ masculino. Su edad está comprendida entre los 26 y 70 años, con una media de 46 . En cuanto al nivel de formación, se ubican así: el $5 \%$ en el nivel de doctorado, el $60 \%$ en maestría, el $30 \%$ en especialización y el $5 \%$ en pregrado. El $19 \%$ de los encuestados alcanza una experiencia docente de 1 a 5 años, mientras que el $21 \%$ está en el rango de
5 a 10 años, el $30 \%$ de 10 a 15 años y el $30 \%$ restante de 15 años en adelante. Respecto al tipo de vinculación, el $87,5 \%$ son profesores catedráticos y el $12,5 \%$ son profesores de planta.

Los docentes, como se observa en la tabla 1, presentan una actitud positiva frente a las TIC. Los de menor edad y menor experiencia presentan una mejor actitud. En los componentes: afectivo, comportamental y cognitivo, se evidencia un alto grado de homogeneidad. Los docentes presentan mejor actitud en el componente afectivo que en el cognitivo y el comportamental, y a su vez, mejor actitud en el cognitivo que en el comportamental. Los docentes con mejor actitud hacia las TIC, presentan más frecuencia de uso de Tl y mayor experticia en TC, para el desarrollo de su labor educativa.

Tabla 1. Correlaciones de las variables estudiadas en los docentes $(\mathrm{N}=80)$.

\begin{tabular}{|c|c|c|c|c|c|c|c|c|}
\hline & & Edad & AEES & ATIC & FUTI & IATI & ETC & IATC \\
\hline \multirow{2}{*}{ Edad } & Cor. Pearson & 1 &, $602^{* *}$ & 018 &,$- 293^{* *}$ & 135 &,$- 408^{* *}$ & 207 \\
\hline & Sig. (bilateral) & &, 000 & ,873 & ,008 & 233 &, 000 & ,065 \\
\hline \multirow{2}{*}{$\begin{array}{l}\text { Años de experien- } \\
\text { cia en educación } \\
\text { superior }\end{array}$} & Cor. Pearson & ,602 & 1 & ,048 &,- 052 & ,078 &,- 156 & ,065 \\
\hline & Sig. (bilateral) &, 000 & & ,675 &, 646 & ,492 & 168 & ,566 \\
\hline \multirow{2}{*}{$\begin{array}{l}\text { Actitud hacia las } \\
\text { TIC }\end{array}$} & Cor. Pearson & ,018 & ,048 & 1 & 178 & ,118 &, $382^{* *}$ & ,025 \\
\hline & Sig. (bilateral) & 873 & 675 & & 115 & 298 &, 000 & 828 \\
\hline \multirow{2}{*}{$\begin{array}{l}\text { Frecuencia de uso } \\
\text { de las TI }\end{array}$} & Cor.Pearson &,$- 293^{* *}$ &,- 052 & 178 & 1 & 138 &, $603^{* *}$ &,- 138 \\
\hline & Sig. (bilateral) & ,008 & ,646 & 115 & & ,223 & ,000 & 221 \\
\hline \multirow{2}{*}{$\begin{array}{l}\text { Interés de aprendi- } \\
\text { zaje en las TI }\end{array}$} & Cor. Pearson & 135 & ,078 & 118 & 138 & 1 & ,076 & $489^{* *}$ \\
\hline & Sig. (bilateral) & 233 & ,492 & 298 & 223 & &, 502 & ,000 \\
\hline \multirow{2}{*}{ Experticia en las TC } & Cor. Pearson &,$- 408^{* *}$ &,- 156 &, $382^{* *}$ &, $603^{* *}$ & ,076 & 1 &,- 071 \\
\hline & Sig. (bilateral) &, 000 & 168 &, 000 & ,000 & ,502 & &, 532 \\
\hline \multirow{2}{*}{$\begin{array}{l}\text { Interés de aprendi- } \\
\text { zaje en las TC }\end{array}$} & Cor. Pearson & 207 & ,065 & ,025 &,- 138 & $489^{* *}$ &,- 071 & 1 \\
\hline & Sig. (bilateral) & ,065 & ,566 & ,828 & 221 & ,000 & ,532 & \\
\hline
\end{tabular}


Los docentes dan cuenta de la importancia del uso de las TIC en la vida cotidiana y el modo en que poco a poco se han ido introduciendo en la academia. De ahí que experimentan con regularidad el uso de nuevos recursos o herramientas digitales en el desarrollo de estrategias de trabajo académico en sus cursos (Ítem 13), y sienten interés por desempeñar un papel de liderazgo en la formación, basada en la innovación y el aprendizaje permanente, enriquecidos por TIC (Ítem 15). Sin embargo, reconocen sus falencias en el dominio de las mismas, de ahí la necesidad de fomento y consolidación de comunidades de aprendizaje y de práctica de los docentes, que permitan a su vez estimular el desarrollo de competencias básicas, intermedias y avanzadas, que tengan en cuenta dimensiones pedagógicas, comunicativas, investigativas y tecnológicas, con relación a la integración de las TIC en el proceso formativo de la modalidad, como se verifica en la tabla 2.

Tabla 2. Percepciones respecto a las TIC y sus niveles de apropiación en los docentes.

\begin{tabular}{|c|c|c|c|}
\hline \multirow{2}{*}{$\begin{array}{l}\text { Docentes entrevistados } \\
\text { Licenciatura en }\end{array}$} & \multicolumn{2}{|c|}{ Percepciones respecto a las TIC } & \multirow{2}{*}{$\begin{array}{l}\text { Niveles de apropiación de TIC } \\
\text { (Hooper y Rieber, 1995) }\end{array}$} \\
\hline & Percepción docente & Importante & \\
\hline Ciencias Naturales y & Grado de utilización & Alto & \multirow[t]{2}{*}{ Integración } \\
\hline Educación Ambiental & Impacto de las TIC & Positivo & \\
\hline Licenciatura en & Percepción docente & Importante & \multirow{3}{*}{ Familiarización } \\
\hline & Grado de utilización & Medio & \\
\hline Literatura y Lengua Castellana & Impacto de las TIC & Positivo & \\
\hline Licenciatura en & Percepción docente & Importante & \multirow{3}{*}{ Integración } \\
\hline & Grado de utilización & Alto & \\
\hline Pedagogía Infantil & Impacto de las TIC & Positivo & \\
\hline Licenciatura en & Percepción docente & Importante & \multirow{3}{*}{ Utilización } \\
\hline & Grado de utilización & Alto & \\
\hline Educación Artística & Impacto de las TIC & Positivo & \\
\hline
\end{tabular}

En cuanto a los estudiantes, de los 193 que respondieron la encuesta, el $78,2 \%$ son de género femenino y el $21,8 \%$ de género masculino, y su edad está comprendida entre los 16 y 54 años, con una media de 27 años. De acuerdo al programa en que se están formando, el 15,5 \% estudian Licenciatura en Ciencias Naturales y Educación Ambiental, el 26,9\% Licenciatura en Educación Artística, el
26,4\% Licenciatura en Literatura y Lengua CasteIlana y el 31,1\% Licenciatura en Pedagogía Infantil. Según el semestre, el $38,8 \%$ de los estudiantes cursan semestres 1 o 2, el 7,8\%, semestres 3 o 4, el $25,4 \%$, semestres 5 o 6 , el 22,8\%, semestres 7 o 8 , y el $5,7 \%$, semestres 9 o 10 . El $88,6 \%$ de los estudiantes afirma tener acceso a computador propio, en tanto que el $11,4 \%$ no lo tiene. Para acceder a 
Internet, el $82,9 \%$ de los estudiantes lo hace en su vivienda, el $9,3 \%$ en un café y el $7,8 \%$ en la Universidad, mediante el móvil o en su entorno laboral.

Los estudiantes presentan una actitud positiva frente a las TIC, la cual está fuertemente correlacionada en forma positiva con la experticia en TIC y medianamente relacionada con la frecuencia de uso de TI. La frecuencia de uso de TI y la experticia en TC están fuertemente correlacionadas con el semestre. En los componentes: afectivo, comportamental y cognitivo, se evidencia un alto grado de homogeneidad entre ellos. En relación con los componentes, los estudiantes presentan mejor actitud en el componente cognitivo que en el comportamental y el afectivo, a su vez, mejor actitud en el comportamental que en el afectivo, aspectos que se evidencian en la tabla 3.

Tabla 3. Correlaciones de las variables estudiadas para los estudiantes $(\mathrm{N}=193)$.

\begin{tabular}{lllllll}
\hline & & Edad & Semestre & ATIC & FTI & ETC \\
\hline \multirow{2}{*}{ Edad } & Cor. Pearson & 1 &, $328^{* *}$ &, 104 &, 008 &,- 083 \\
& Sig. (bilateral) & &, 000 &, 149 &, 913 &, 251 \\
Semestre & Cor. Pearson &, $328^{* *}$ & 1 &, 125 &, $235^{* *}$ &, $156^{*}$ \\
& Sig. (bilateral) &, 000 & &, 084 &, 001 &, 030 \\
Actitud hacia las TIC & Cor. Pearson &, 104 &, 125 & 1 &, $163^{*}$ &, $266^{* *}$ \\
& Sig. (bilateral) &, 149 &, 084 & &, 024 &, 000 \\
Frecuencia de uso de las TI & Cor. Pearson &, 008 &, $235^{* *}$ &, $163^{*}$ & 1 &, $575^{* *}$ \\
& Sig. (bilateral) &, 913 &, 001 &, 024 & &, 000 \\
Experticia en las TC & Cor. Pearson &,- 083 &, $156^{*}$ &, $266^{* *}$ &, $575^{* *}$ & 1 \\
& Sig. (bilateral) &, 251 &, 030 &, 000 &, 000 & \\
& $* * *$ La correlación es significativa en el nivel 0,01 (bilateral). & & \\
& $*$. La correlación es significativa en el nivel 0,05 (bilateral). & & \\
\hline
\end{tabular}

Respecto al uso de las TIC por parte de los docentes, éste se ve menguado en la medida en que ellos, según la percepción de los estudiantes, dan mayor relevancia al trabajo orientado presencial. Ratificándose, entonces, la necesidad de fortalecer la comunicación entre los estudiantes, así como el seguimiento y el acompañamiento requerido por ellos, lo que corresponde al trabajo orientado no presencial y al trabajo independiente, donde las TIC pueden jugar un papel trascendental. En este sentido, como lo expresan los estudiantes, ellos asumen el reto y la posibilidad de integración de
TIC en su proceso de formación, al igual que en el futuro de su práctica docente, frente a su forma de actuar y pensar en su proceso formativo en la modalidad.

Las directoras de los programas de FID que se tomaron como referencia para la investigación, si bien tienen una percepción Importante, de acuerdo con Riascos, Quintero y Ávila (2009), reconocen que el tema de las TIC tiene una presencia mínima en los planes de estudio, limitándose a los cursos cuyo contenido tiene que ver directamente con el 
área, y en algunos casos hace parte de otro tipo de cursos: por ejemplo, "en el curso de investigación formativa de cuarto semestre [los estudiantes reciben] una capacitación sobre uso de bases de datos y buscadores. Es el ejercicio que hacen" (Directora LLLC, comunicación personal, 2017).

Lo anterior indica que, según las directoras, es casi un axioma que las TIC funcionan como un complemento de las actividades desarrolladas en el aula, por lo que no se les otorga la posibilidad de ser pensadas como un escenario legítimo de aprendizaje, tanto como una clase de tipo magistral. Así mismo, las barreras para su uso e implementación, no son solo de tipo generacional o cultural, sino disciplinares. Si un docente considera que las TIC, respecto del área que trabaja, no resultan una práctica funcional, y es más, constituye una vulneración de su ethos profesional, se abstiene, evita e incluso sataniza el uso de TIC en el aula.

3.2 Experiencia de construcción de entornos digitales de aprendizaje

En el planteamiento de los ambientes digitales de aprendizaje, se tuvieron en cuenta las directrices ofrecidas en la Estrategia de formación en la modalidad de educación a distancia de la Universidad del Tolima (2015). En ellas se hacen algunas precisiones epistemológicas sobre el diseño y la estructura curricular de la pedagogía problémica, así: se concibe que el profesor tiene el rol de orientador o guía y, en consecuencia, los estudiantes asumen la responsabilidad de aprender; los problemas no son tomados de forma aislada ni descontextualizada, sino que, por el contrario, parten de la realidad misma previamente analizada y leída por el docente o quienes han diseñado el programa. El problema se convierte en un reto académico para los estudiantes, pues éste los motiva y anima para que participen activa y colaborativamente en su resolución. Además, al final del ejercicio, siempre será importante que los estudiantes evalúen sus procesos individual y grupal.
Otro elemento importante que se tuvo en cuenta fueron los fundamentos teóricos sobre los procesos de enseñanza y aprendizaje en entornos virtuales, desde una perspectiva constructivista y sociocultural, como lo plantean Coll, Mauri y Onrubia (2009). En el caso de la modalidad de educación a distancia de la Universidad del Tolima, esta se orienta en la perspectiva de ambientes híbridos de aprendizaje, en tanto permite la articulación del trabajo independiente del estudiante y el trabajo de acompañamiento del profesor, presencial y en línea (Ramírez \& Solano, 2017).

En las narraciones de las experiencias vividas por las docentes de los cursos Lenguaje, cognición y sociedad, y Filosofía de la educación, coinciden en señalar que el docente diseñador del portafolio pedagógico, se enfrenta a un proceso que requiere de disposición para mirar de otra manera los procesos de enseñanza y de aprendizaje mediados por las TIC, en la modalidad de educación a distancia, y así apropiar esta estrategia. En este sentido, ambas hacen énfasis en la plena conciencia que se debe tener sobre la educación a distancia y dentro de ella de categorías que orientan la formación en el IDEAD de la Universidad del Tolima, a fin de que la propuesta de formación sea coherente y pertinente $y$, de este modo, se facilite el trabajo académico de los estudiantes y de los profesores.

Una vez diseñados los portafolios de los cursos mencionados anteriormente, se valoraron teniendo en cuenta las dimensiones: pedagógica, comunicativa y tecnológica del diseño, de una parte, y de otra, los componentes de la estrategia de formación: currículo, pedagogía y didáctica, metodología, evaluación, y medios, del IDEAD. Estos criterios constituyeron las afirmaciones de un instrumento a partir de una escala Likert, que fue complementado con preguntas abiertas sobre la experiencia. El instrumento se aplicó a 22 estudiantes: 13 del curso Filosofía de la Educación (Grupo 1), y 9 del curso Lenguaje, cognición y sociedad (Grupo 2). Los estudiantes cursaban el semestre siguiente al que corresponde cada uno de los cursos; es decir, habían aprobado el respecti- 
vo curso. Para efectuar la valoración, se desarrolló una sesión inicial de trabajo en la que se les solicitó explorar el entorno digital desarrollado para cada curso y, en particular, una unidad de contenido.

En líneas generales, la percepción fue positiva para la mayoría de los estudiantes con respecto al portafolio pedagógico, considerándolo como una herramienta que contribuye a la formación y el aprendizaje autónomo, dos aspectos esenciales de la educación a distancia. Sin embargo, se resalta la importancia de un aprendizaje previo en el que se garantice el entrenamiento para el manejo de la plataforma y el conocimiento de lo que son los portafolios pedagógicos. No obstante, vale la pena resaltar que en algunos estudiantes se percibe su comodidad por el trabajo tradicional en el aula, expresado en afirmaciones como la siguiente: "No me gusta mucho la idea del ambiente digital, ya que después de tanto tiempo sin manipularlo uno se acostumbra a otros medios" (Est. Semestre VII, Grupo 1). Finalmente, algunos aprovecharon el espacio para manifestar el desconocimiento frente a los recursos y la utilización de la propuesta pedagógica en ambiente digital:"Bueno, pero le falta un poco de asesoramiento para un manejo más eficaz y contribuyente en el mismo" (Est. Semestre VII, Grupo 1).

Los resultados indican que la experiencia de diseño de portafolios pedagógicos para el Instituto de Educación a Distancia, IDEAD, podrá ser aún más enriquecedora si se logran entretejer, a través de un proceso dinámico, la interacción de los docentes en un trabajo en red con los que pertenecen a las mismas áreas de formación, o que orientan el mismo curso a nivel nacional. La experiencia también se podrá mejorar si se someten los resultados del diseño, a la evaluación de pares diseñadores de portafolios pedagógicos.

\section{Conclusiones}

Los procesos de formación trascienden los ejercicios instructivos y de capacitación, en tanto que pretenden una transformación del sujeto que se forma y buscan su deslocalización para que encuentre o construya en un nuevo terreno, su propio escenario. En este sentido, a través del desarrollo del taller de diseño de portafolios pedagógicos, se logró comprender que el docente diseñador de portafolios pedagógicos va reconstituyendo su quehacer pedagógico en la medida en que va creando su obra. Se trata de una obra creadora que lo recrea como maestro, gracias a la permanente problematización y reconceptualización de su saber y de las pretensiones formativas, ejercicio que, a su vez, lo va llevando paulatinamente a la idea de que este mismo sendero sea el camino que debe seguir su estudiante como maestro en formación.

La experiencia de desarrollo de portafolios pedagógicos trasciende la lógica de la ejecución procedimental, pues si bien es necesario el diligenciamiento de los formatos establecidos, el acto diseño del portafolio pedagógico es un proceso de invención educativa. Esto debido a que implica el acto creativo de generar dinámicas auténticas de diseño pedagógico, tecnológico y comunicativo, que tienen como punto de partida el camino ganado por los docentes, pero que debe conjugar las potencialidades de los estudiantes con las problemáticas, los contenidos y las necesidades de formación, para dar respuesta a las urgencias académicas y sociales de un contexto determinado.

La valoración de los portafolios hecha por los estudiantes, revela que su construcción implica una necesaria disposición inicial frente al uso y la apropiación de las TIC, que permita tener un acercamiento positivo a la incorporación del portafolio pedagógico, así como su aporte a la educación a distancia. Lo anterior teniendo en cuenta que la mayor parte de los estudiantes, estuvieron de acuerdo con cada una de las afirmaciones planteadas, frente a las tres dimensiones: pedagógica, comunicativa y tecnológica, así como con los cinco componentes evaluados: curricular, didáctico, metodológico, evaluación y medios, respecto al diseño de los portafolios. 
Teniendo en cuenta que la valoración que se hizo correspondió a los materiales y al entorno digital, sería importante poder contrastarlas con una experiencia completa de formación del curso durante un semestre lectivo, en el que docentes y estudiantes puedan manifestar su sentir con la utilización del mismo, respecto de las dimensiones del portafolio.

Finalmente, se hace necesario que los lineamientos que iluminaron la experiencia sean desarrollados en un nivel mayor de detalle, de tal manera que permitan descender a niveles más bajos del proceso, como la elaboración de guías u orientaciones precisas que den pie a instrumentos para las valoraciones de los portafolios pedagógicos, de los objetos y, en general, de todo tipo de material que se produzca en el marco de los diseños pedagógicos de formación.

\section{Agradecimientos}

Hacemos un reconocimiento a la contribución de: July Lizeth Bolívar Rodríguez, Comunicadora Social - Periodista, Magíster en Educación; Paula Leal Pérez, Profesional en Ciencias Sociales; y a John James Otavo Velázquez, Profesional en Matemáticas y Estadística.

\section{Referencias}

Álvarez, E. E. (2015). Estándares y competencias TIC de la dimensión técnica para la formación inicial docente. Estudio correlacional en estudiantes de seis carreras de pedagogía de la Universidad de Antofagasta (Tesis de maestría). Universidad de Chile, Santiago, Chile. Recuperado de: http://repositorio. uchile.cl/handle/2250/136542

Álvarez, G., \& Morán, L. (2014). ¿Cómo se dispone a los docentes para futuras prácticas con tecnologías?. Revista de Educación a Distancia, 43. Recuperado de: http://www.um.es/ead/red/43

Arias, R. A., \& Vanegas, H. (2013). Caracterización de los procesos de implementación y uso de las aulas virtuales en la Facultad de Educación. Sánchez, J. (Ed.). Nuevas ideas en informática educativa. Memorias del XVII Congreso Internacional de Informática Educativa, TISE. Santiago, Chile: Universidad de Chile. Recuperado de: http://www.tise.cl/ volumen9/TISE2013/

Ballesta, J., \& Céspedes, R. (2015). Los contenidos de Tecnología Educativa en las titulaciones de grado de las universidades españolas. Revista Latinoamericana de Tecnología Educativa, 14 (1), 133-143. doi: 10.17398/1695288X.14.1.17.

Brun, M. (2011). Las tecnologías de la información y las comunicaciones en la formación inicial docente en América Latina y el Caribe. Santiago, Chile: Organización de las Naciones Unidas. Recuperado de: http://repositorio.cepal.org/bitstream/11362/6183/1/S1100626_es.pdf

Cifuentes, G., \& Cerda, C. (2013). Distancia entre percepción de uso de TIC y uso efectivo de campus virtual en académicos formadores de profesores. Revista Educación y Tecnología, 3, 46-60. Recuperado de: http://revistas.umce.cl/index.php/edytec/ article/view/135

Coll, C., Mauri, T., \& Onrubia, J. (2008). Los entornos virtuales de aprendizaje basados en el análisis de casos y la resolución de problemas. En C. Coll \& C. Monereo (Eds.), Psicología de la educación virtual. Aprender y enseñar con las tecnologías de la información y la comunicación. Madrid, España: Morata.

Cózar, R., \& Roblizo, M. J. (2014). La competencia digital en la formación de los futuros maestros: percepciones de los alumnos de los Grados de Maestro de la Facultad de Educación de Albacete. Revista Latinoamericana de Tecnología Educativa, 13 (2), 119-133. doi: 10.17398/1695288X.13.2.119

Cruz-Rojas, G. A., Molina-Blandón. M. A., \& Valdiri-Vinasco. V. (2019). Vigilancia tecnológica para la innovación educativa en el uso de bases de datos y plataformas de gestión de aprendizaje en la 
universidad del Valle, Colombia. Revista de Investigación, Desarrollo e Innovación, 9 (2), 303-317. doi: 10.19053/20278306.v9.n2.2019.9175

Díez, E. J. (2012). Modelos socioconstructivistas y colaborativos en el uso de las TIC en la formación inicial del profesorado. Revista de Educación, 358, 175-196. Recuperado de: http://www.revistaeducacion.educacion.es/re358/re358_09.pdf

Dussel, I. (ed.). (2014). Incorporación con sentido pedagógico de TIC en la formación docente de los países del Mercosur. Buenos Aires, Argentina:Teseo. Recuperado de: http://www.pasem.org/IMG/pdf/14.pdf

Elliott, J. (2010). La investigación-acción en educación. Madrid, España: Morata.

Fraga, F., \& Gewerc, A. (2015) Creencias sobre Tecnología Educativa: una mirada desde la biografía escolar y universitaria de una maestra en formación inicial. Revista Latinoamericana de Tecnología Educativa, 14 (3), 23-34. doi: 10.17398/1695288X.14.3.23

Garnica-Hoz, J. L. (2015). Nuevos "ambientes de aprendizaje" para viejas prácticas pedagógicas: el uso de Moodle en la Facultad de Educación de la Universidad de Cantabria (Trabajo de Máster). Universidad Nacional de Educación a Distancia, Madrid, España. Recuperado de: http://e-spacio.uned.es/ fez/view/bibliuned:masterComEdred-Jlgarnica

Garrido, J. M. (2009). Creencias sobre el rol de las tecnologías de información y comunicación en la formación inicial de docentes: explorando las diferencias entre estudiantes y docentes universitarios (Tesis doctoral). Universidad de Barcelona, Barcelona, España. Recuperado de: http://diposit. ub.edu/dspace/handle/2445/41486

Gewerc, A., \& Montero, L. (2015). Conocimiento profesional y competencia digital en la formación del profesorado. El caso del Grado de Maestro en Educación Primaria. Revista Latinoamericana de Tecnología Educativa, 14 (1), 31-43. doi: 10.17398/1695288X.14.1.17

Guisao-Gil, G. S. (2011). Percepción de docentes y estudiantes en relación con el uso de las TIC en los procesos de enseñanza-aprendizaje. Estudio realizado en la Licenciatura en Educación Básica con Énfasis en Humanidades y Lengua Castellana, del Tecnológico de Antioquia Institución Universitaria, en el año 2009 (Tesis de maestría). Universidad de Medellín, Medellín, Colombia. Recuperado de: http:// repository.udem.edu.co/handle/11407/84

Gisbert-Cervera, M., González-Martínez, J., \& Esteve-Mon, F. (2016). Competencia digital y competencia digital docente: una panorámica sobre el estado de la cuestión. Revista Interuniversitaria de Investigación en Tecnología Educativa, 0, 74-83. doi: https://doi.org/10.6018/riite2016/257631

Hepp, P., Marés, L., \& Severin, E. (2012). Caracterización de buenas prácticas en formación inicial docente en TIC. Buenos Aires, Argentina: Organización de Estados Americanos.

Hinojosa, V., Valdez, L., Servín, S., \& Perusquía, E. (2015). La incorporación de las TIC en la práctica docente de los profesores del Sistema Universidad Abierta y Educación a Distancia de la Universidad Nacional Autónoma de México: Catálogo CUAED de herramientas colaborativas. XVI Encuentro Internacional Virtual Educa México. Guadalajara, México: Organización de los Estados Americanos y Gobierno del Estado de Jalisco. Recuperado de: http://www.virtualeduca.pro/documentos/23/IncorporacioonTICpracticaDocenteCatalogoCUAED. pdf

Hooper, S., \& Rieber, L. P. (1995). Teaching with Technology. En A. C. Ornstein (Ed.), Teaching: Theory into practice, 154-170. Needham Heights, USA: Allyn and Bacon. Recuperado de http://www.d11. org/NextGen/Documents/Hooper\%20and\%20 Rieber.pdf 
Laura, C. D., Sosa, O. E., \& Almanza, L. A. (2015). Formación inicial docente y tecnologías: ¿cuáles son los niveles de integración de TIC en las prácticas pedagógicas universitarias? X Congreso sobre Tecnología en Educación \& Educación en Tecnología (TE \& ET). Corrientes, Argentina: Red de Universidades con Carreras en Informática. Recuperado de: http://sedici.unlp.edu.ar/handle/10915/48726

Leite, C., Martínez, R., \& Monteiro, A. (2016). TIC y formación inicial de maestros: oportunidades y problemas desde la perspectiva de estudiantes. Cuadernos de Investigación Educativa, 7 (1), 69-92. doi:http://dx.doi.org/10.18861/cied.2016.7.1.2577

Mellado, M. E. (2010). Portafolio en línea en la formación inicial docente. Revista Electrónica de Investigación Educativa, 12 (1). Recuperado de: http:// redie.uabc.mx/vol12no1/contenido-melladohdez. html

Ministerio de Educación Nacional (2013). Competencias TIC para el desarrollo profesional docente. Bogotá, Colombia.

Occelli, M., García, L., \& Masullo, M. (2012). Integración de las TICs en la formación inicial de docentes y en sus prácticas educativas. Virtualidad, Educación y Ciencia, 3 (5), 59-72. Recuperado de: https://revistas.unc.edu.ar/index.php/vesc/article/view/3013

Parra, L. R., \& Cardona, C. E. (2013). Usos pedagógicos de las tecnologías de la información y la comunicación: en prácticas educativas formales en la educación superior. XII Foro de Investigadores de Informática Educativa Versión Internacional. Medellín, Colombia: Red Iberoamericana de Informática Educativa.

Ramírez, J. E., \& Solano, J. C. (2017). Ambientes digitales de aprendizaje: una aproximación pedagógica, tecnológica y comunicativa para la educación a distancia en la Universidad del Tolima. XIII
Congreso de Informática Educativa Versión Internacional, Montería, Colombia.

Riascos, S. C., Quintero, D. M., \& Ávila, G. P. (2009). Las TIC en el aula: percepciones de los profesores universitarios. Educación y Educadores, 12 (3), 133157. Recuperado de: http://www.redalyc.org/articulo.oa?id=83412235008

Rozo-Sandoval, A. C., \& Prada-Dussán, M. (2012). Panorama de la formación inicial docente y TIC en la región andina. Revista Educación y Pedagogía, 24 (62), 191-204. Recuperado de: http://aprendeenlinea.udea.edu.co/revistas/index.php/revistaeyp/ article/view/14203

Salinas, J. (2012). La investigación ante los desafíos de los escenarios de aprendizaje futuros. Revista de Educación a Distancia, 32. Recuperado de: http:// revistas.um.es/red/article/view/233091

Sánchez, J., \& Morales, S. (2012). Docencia universitaria con apoyo de entornos virtuales de aprendizaje (EVA). Digital Education Review, 21, 33-46. Recuperado de: http://greav.ub.edu/der

Sancho, J. M., Bosco, A., Alonso, C., \& Sánchez, J. A. (2015). Formación del profesorado en Tecnología Educativa: de cómo las realidades generan los mitos. Revista Latinoamericana de Tecnología Educativa, 14 (1), 17-30. doi: 10.17398/1695288X.14.1.17

Silva-Quiroz, J. E., \& Astudillo-Cavieres, A. V. (2012). Inserción de TIC en la formación inicial docente: barreras y oportunidades. Revista Iberoamericana de Educación, 58 (4). Recuperado de: http://www. rieoei.org/deloslectores/4557Silva.pdf

Tobón, M. I., Arbeláez, M. C., Falcón, M. C., \& Bedoya, J. R. (2010). La formación docente al incorporar las TIC en los procesos de enseñanza y aprendizaje. Una propuesta para la Universidad Tecnológica de Pereira. Pereira, Colombia: Universidad Tecnológica de Pereira. Disponible en: https://univirtual.utp. 
edu.co/portal/archivos_nota/165316_laformaciondocente.pdf

Universidad del Tolima (2015). Proyecto educativo institucional. Ibagué, Colombia.

Vaillant, D. (2013). Integración de TIC en los sistemas de formación docente inicial y continua para la
Educación Básica en América Latina. Buenos Aires, Argentina: Fondo de las Naciones Unidas para la Infancia. Recupperado de: https://www.unicef. org/argentina/spanish/educacion_Integracion_ TIC_sistemas_formacion_docente.pdf

Valles, M. S. (2007). Entrevistas cualitativas. Madrid, España: Centro de Investigaciones Sociológicas. 
